\title{
Imperfect interfaces with graded materials and unilateral conditions: theoretical and numerical study
}

\author{
Serge Dumont \\ Université de Nîmes, MIPA, IMAG, Montpellier, France
}

Frédéric Lebon

Aix-Marseille University, CNRS, Centrale Marseille, LMA, Marseille, France

\author{
Raffaella Rizzoni \\ Dipartimento di Ingegneria, Università di Ferrara, Ferrara, Italy
}

\begin{abstract}
In this paper a composite body is considered. This body is made of three solids: two linear elastic adherents and a piecewise linear thin adhesive. The composite occupies a bounded domain depending naturally on a small parameter $\varepsilon$, which is the thickness, assumed constant, of the adhesive. Classically, it is possible to derive an interface imperfect law using asymptotic expansions as the thickness $\varepsilon$ tends to zero. In this work, the material in the interphase is assumed to be graded, i.e. its elasticity properties vary along the thickness. Moreover, an unilateral condition is considered to avoid penetrations. A first result of the paper is that it is possible to apply the above methodology based on asymptotic expansions to this kind of material. Then, a finite element method is introduced to solve the initial problem (with three layers) and the limit problem (with two layers in imperfect contact). Various types of graded materials are numerically analyzed. In particular, different types of stiffness distributions are studied in detail.
\end{abstract}

\section{Keywords}

Interfaces, micromechanics, asymptotic expansions, contact mechanics, elasticity

\section{Introduction}

Functionally graded materials (FGMs) are characterized by smooth variations of thermal, physical, chemical, and mechanical properties [1], usually in a specific direction. These variations are due to gradual changes in the composition, morphology, and crystalline structure over volume. This kind of inhomogeneous revolutionary material can be designed for particular, and often unique, function or applications. The overall properties of a FGM are different from any of the constituents that form it. Nowadays, there is a wide range of real applications. Even if FGMs are typical in natural material (bone, teeth, etc.), the first technological development is due to Japanese researchers, who had the challenge of creating a material capable of bearing a thermal gradient of $1000^{\circ} \mathrm{C}$ on a thickness of only $10 \mathrm{~mm}$ [2]. It is at present in the nuclear sector (thermonuclear fusion) that

Corresponding author:

S. Dumont, Université de Nîmes, IMAG - CNRS UMR 5I49, Place E. Bataillon, 34095 Montpellier Cedex 5, France.

Email: serge.dumont@unimes.fr 


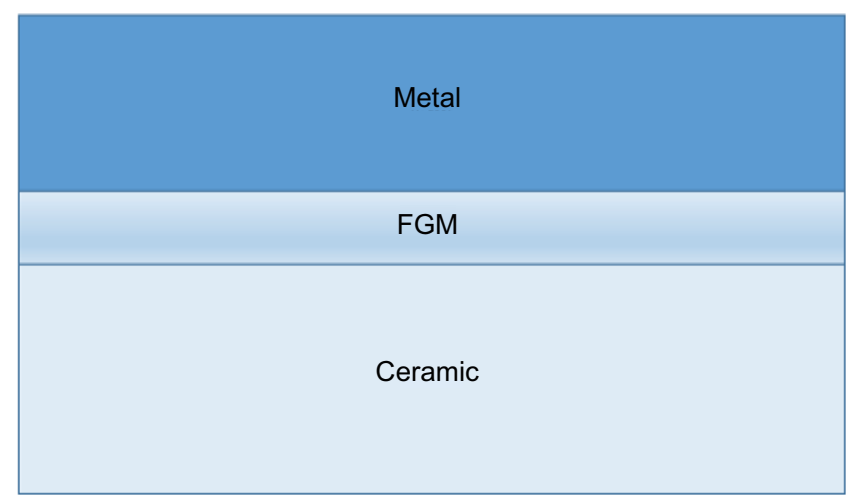

Figure I. Example of a FGM application: adhesive bonding of two basic constituents with specific thermic and mechanical properties

the need for FGMs is greatest. Specifications (tenacity, heat resistance, resistance to corrosion, or irradiation) become increasingly binding, often leading to contradictory requests that a unique monolithic material cannot perform. As an example, certain types of steels are characterized by a conflict between the thermal resistance and the tenacity. Owing to these requirements and as a supplement to the usual strategies of microstructure optimization, optimal designs based on material structuring at the mesoscopic scale have also been explored. Other structural applications require a joint between two basic constituents, for example ceramic and metal (see Figure 1). This class of FGMs offers many advantages; in particular, the ceramic face provides high resistance to wear, while the opposite metal face offers high hardness and mechanical strength. Such materials are expected to be very desirable for tribological applications, where wear resistance and high hardness are required simultaneously.

In this paper, we are interested in modeling the behavior of FGM thin films, whose thickness is very small with respect to that of the adherents (metal and ceramic, for example). The thickness of the FGM is considered as a small parameter and the limit problem is analyzed as the thickness tends to zero. In the present paper, a simple case is considered: the stiffness of the FGM linearly rescales with its thickness (soft material hypothesis). The limit problem is derived using matched asymptotic expansions [3-8] and the obtained limit condition turns to be a law of imperfect interface. Here, the term perfect interface means that the jumps in the displacement and in the traction vectors across the interface are equal to zero. This situation arises, for example, when the rigidity of the interphase is of the same order of the rigidity of the adherents. In contrast, the term imperfect interface means that either the jumps in the displacement or the jumps in the traction vectors are not vanishing. This last type of interface has been widely studied in previous works and in several applications (see, for example, [9-12]).

The rest of this paper is divided into four sections. Section 2 is devoted to the mechanical problem and to introducing some notation. In Section 3, an asymptotic analysis is performed to obtain a law of imperfect interface. The finite element implementation is presented in Section 4. Section 5 focuses on the numerical study of some academic examples.

\section{The mechanical problem}

Let us now consider the adhesive bonding of two linear elastic three-dimensional bodies, where the mechanical properties of the adhesive may vary along the thickness (the third direction).

For that purpose, we define the following domains:

- $\Omega \subset \mathbb{R}^{3}$ is the whole structure, of boundary $\partial \Omega$;

- $B^{\varepsilon}=\left\{\left(x_{1}, x_{2}, x_{3}\right) \in \Omega:\left|x_{3}\right|<\frac{\varepsilon}{2}\right\}$ is the glue, also called the interphase;

- $\quad \Omega_{ \pm}^{\varepsilon}=\left\{\left(x_{1}, x_{2}, x_{3}\right) \in \Omega: \pm x_{3}>\frac{\varepsilon}{2}\right\}$ are the adherents;

- $S_{ \pm}^{\varepsilon}=\left\{\left(x_{1}, x_{2}, x_{3}\right) \in \Omega: x_{3}= \pm \frac{\varepsilon}{2}\right\}$ are the surfaces between the adhesive and the adherents;

- $\quad \Gamma=\left\{\left(x_{1}, x_{2}, x_{3}\right) \in \Omega: x_{3}=0\right\}$ is the interface, the geometrical limit of $B^{\varepsilon}$ as $\varepsilon$ vanishes;

- $B=\left\{\left(x_{1}, x_{2}, x_{3}\right) \in \Omega:\left|x_{3}\right|<\frac{1}{2}\right\}$ is the rescaled interphase;

- $\Omega_{ \pm}=\left\{\left(x_{1}, x_{2}, x_{3}\right) \in \Omega: \pm x_{3}>\frac{1}{2}\right\}$ are the adherents in the rescaled configuration; 
- $S_{ \pm}=\left\{\left(x_{1}, x_{2}, x_{3}\right) \in \Omega: x_{ \pm}= \pm \frac{1}{2}\right\}$ are the surfaces between the adhesive and the adherents in the rescaled configuration;

- $\Omega_{ \pm}^{0}=\left\{\left(x_{1}, x_{2}, x_{3}\right) \in \Omega: \pm x_{3}>0\right\}$ are the domains occupied by the adherents in the limit configuration obtained as $\varepsilon$ vanishes.

On a part $\Gamma_{1}$ of $\partial \Omega$, an external load $g$ is applied, and on $\Gamma_{0} \subset \partial \Omega$, such that $\Gamma_{0} \cap \Gamma_{1}=\emptyset$, a displacement $u_{d}$ is imposed. Moreover, we suppose that $\Gamma_{0} \cap B^{\varepsilon}=\emptyset$ and $\Gamma_{1} \cap B^{\varepsilon}=\emptyset$. A body force $f$ is applied in $\Omega_{ \pm}^{\varepsilon}$. We consider also that the interface $\Gamma$ is a plane normal to the third direction $e_{3}$. We are interested in the equilibrium of such a structure.

The equations of the mechanical problem are

$$
\begin{cases}\operatorname{div} \sigma^{\varepsilon}+f=0 & \text { in } \Omega_{ \pm}^{\varepsilon} \cup B^{\varepsilon} \\ \sigma^{\varepsilon} n=g & \text { on } \Gamma_{1} \\ u^{\varepsilon}=u_{d} & \text { on } \Gamma_{0} \\ \sigma^{\varepsilon}=A_{ \pm} e\left(u^{\varepsilon}\right) & \text { in } \Omega_{ \pm}^{\varepsilon} \\ \sigma^{\varepsilon}=A_{i}\left(e\left(u^{\varepsilon}\right)\right) & \text { in } B^{\varepsilon}\end{cases}
$$

where $e\left(u^{\varepsilon}\right)$ is the strain tensor $\left(e_{i j}\left(u^{\varepsilon}\right)=\frac{1}{2}\left(u_{i, j}+u_{j, i}\right), i, j=1,2,3\right)$ and $A_{ \pm}$are the elasticity tensors of the deformable adherents. Moreover, $u^{\varepsilon}$ and $\sigma^{\varepsilon}$ are considered as continuous across the interfaces $S_{+}^{\varepsilon}$ and $S_{-}^{\varepsilon}$. The piecewise linear operator $A_{i}$, which defines the constitutive equation of the adhesive interphase, is defined in the following. In the interphase $B^{\varepsilon}$, two isotropic regimes (traction vs compression) are considered:

$$
\left\{\begin{array}{l}
\sigma^{\varepsilon}=\hat{\lambda} \operatorname{tr}\left(e\left(u^{\varepsilon}\right)\right) I d+2 \hat{\mu} e\left(u^{\varepsilon}\right) \text { if } \operatorname{tr}\left(e\left(u^{\varepsilon}\right)\right) \geq 0 \\
\sigma^{\varepsilon}=\bar{\lambda} \operatorname{tr}\left(e\left(u^{\varepsilon}\right)\right) I d+2 \hat{\mu} e\left(u^{\varepsilon}\right) \text { if } \operatorname{tr}\left(e\left(u^{\varepsilon}\right)\right) \leq 0
\end{array}\right.
$$

Let us emphasize that the strictly positive Lamé's coefficients of the interphase depend on the thickness $\varepsilon$ of the interphase, i.e. we consider in this study that

$$
\hat{\lambda}=\lambda\left(x_{3}\right) \varepsilon, \quad \hat{\mu}=\mu\left(x_{3}\right) \varepsilon, \quad \bar{\lambda}=\bar{\lambda}\left(x_{3}\right)
$$

As a consequence, in this study the adhesive has a non-symmetric behavior in traction (soft) and in compression (hard).

\section{Asymptotic analysis}

The thickness of the interphase being very small, we seek the solution of problem (1) using asymptotic expansions with respect to the parameter $\varepsilon$ :

$$
\left\{\begin{array}{l}
u^{\varepsilon}=u^{0}+\varepsilon u^{1}+O\left(\varepsilon^{2}\right) \\
\sigma^{\varepsilon}=\sigma^{0}+\varepsilon \sigma^{1}+O\left(\varepsilon^{2}\right)
\end{array}\right.
$$

At this level, in order to work in a configuration independent of $\varepsilon$, the domain is rescaled using the classical procedure:

- In the glue, we define the following change of variable

$$
\begin{aligned}
& \left(x_{1}, x_{2}, x_{3}\right) \in B^{\varepsilon} \rightarrow\left(z_{1}, z_{2}, z_{3}\right) \in B, \\
& \text { with }\left(z_{1}, z_{2}, z_{3}\right)=\left(x_{1}, x_{2}, \frac{x_{3}}{\varepsilon}\right)
\end{aligned}
$$

(see configuration 2 of Figure 2) and we denote $\hat{u}^{\varepsilon}\left(z_{1}, z_{2}, z_{3}\right)=u^{\varepsilon}\left(x_{1}, x_{2}, x_{3}\right)$ and $\hat{\sigma}^{\varepsilon}\left(z_{1}, z_{2}, z_{3}\right)=$ $\sigma^{\varepsilon}\left(x_{1}, x_{2}, x_{3}\right)$.

- In the adherents, we define the following change of variables

$$
\begin{aligned}
& \left(x_{1}, x_{2}, x_{3}\right) \in \Omega_{+}^{\varepsilon} \rightarrow\left(z_{1}, z_{2}, z_{3}\right) \in \Omega_{+}, \\
& \text {with }\left(z_{1}, z_{2}, z_{3}\right)=\left(x_{1}, x_{2}, x_{3}+1 / 2-\varepsilon / 2\right)
\end{aligned}
$$




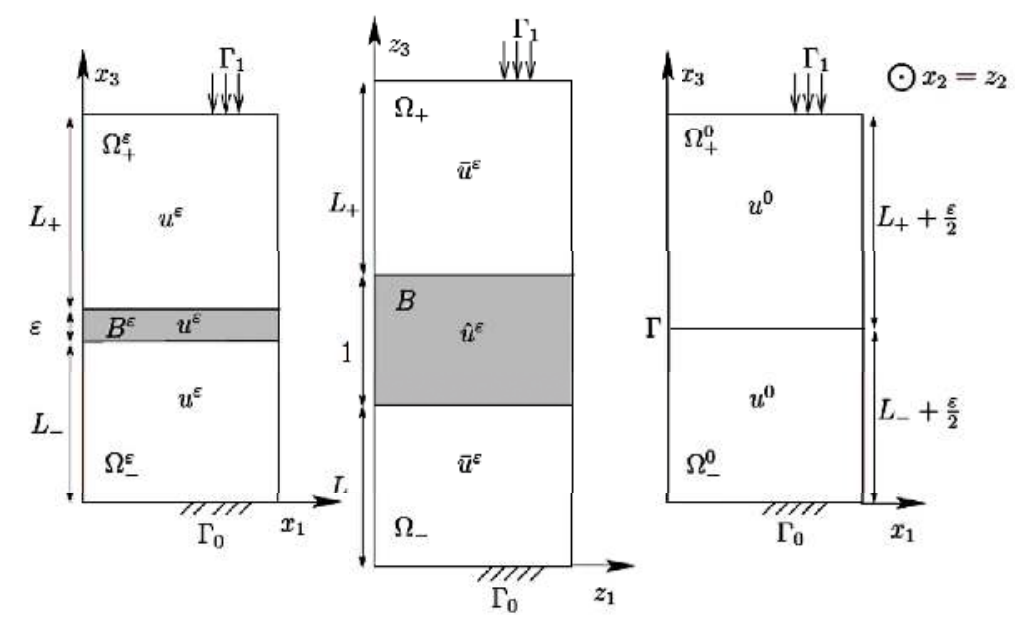

Figure 2. Geometry of the interphase/interface problem. Left: the initial problem with an interphase of thickness $\varepsilon$. Middle: the rescaled problem with interphase height equal to I $\mathrm{m}$ (having length dimension) in which the asymptotical analysis is realized. Right: the limit interface problem.

and

$$
\begin{aligned}
& \left(x_{1}, x_{2}, x_{3}\right) \in \Omega_{-}^{\varepsilon} \rightarrow\left(z_{1}, z_{2}, z_{3}\right) \in \Omega_{-}, \\
& \text {with }\left(z_{1}, z_{2}, z_{3}\right)=\left(x_{1}, x_{2}, x_{3}-1 / 2+\varepsilon / 2\right)
\end{aligned}
$$

We denote $\bar{u}^{\varepsilon}\left(z_{1}, z_{2}, z_{3}\right)=u^{\varepsilon}\left(x_{1}, x_{2}, x_{3}\right)$ and $\bar{\sigma}^{\varepsilon}\left(z_{1}, z_{2}, z_{3}\right)=\sigma^{\varepsilon}\left(x_{1}, x_{2}, x_{3}\right)$. We suppose that the external forces and the prescribed displacement $u_{d}$ are assumed to be independent of $\varepsilon$. As a consequence, we define $\bar{f}\left(z_{1}, z_{2}, z_{3}\right)=f\left(x_{1}, x_{2}, x_{3}\right), \bar{g}\left(z_{1}, z_{2}, z_{3}\right)=g\left(x_{1}, x_{2}, x_{3}\right)$ and $\bar{u}_{d}\left(z_{1}, z_{2}, z_{3}\right)=u_{d}\left(x_{1}, x_{2}, x_{3}\right)$.

- The classical matching at the interface adherent/adhesive is

$$
\begin{aligned}
& u^{0}\left(x_{1}, x_{2}, 0^{ \pm}\right) \approx u^{0}\left(x_{1}, x_{2}, \pm \varepsilon / 2\right)=\hat{u}^{0}\left(x_{1}, x_{2}, \pm 1 / 2\right)=\bar{u}^{0}\left(x_{1}, x_{2}, \pm 1 / 2\right) \\
& \sigma^{0}\left(x_{1}, x_{2}, 0^{ \pm}\right) \approx \sigma^{0}\left(x_{1}, x_{2}, \pm \varepsilon / 2\right)=\hat{\sigma}^{0}\left(x_{1}, x_{2}, \pm 1 / 2\right)=\bar{\sigma}^{0}\left(x_{1}, x_{2}, \pm 1 / 2\right)
\end{aligned}
$$

From the equilibrium equation in the adhesive (1-1) it follows that

$$
\hat{\sigma}_{i j, j}^{\varepsilon}=0
$$

and using (4), the $\frac{1}{\varepsilon}$ term in the development is identified as

$$
\hat{\sigma}_{i 3,3}^{0}=0
$$

leading to

$$
\left[\left[\hat{\sigma}_{i 3}^{0}\right]\right]=0
$$

and

$$
\hat{\sigma}_{i \alpha, \alpha}^{0}=0
$$

for $i=1,2,3$ and $\alpha=1,2$, where for a given function $h$, the following notation has been introduced: [[h]] $=$ $h\left(z_{1}, z_{2},+\frac{1}{2}\right)-h\left(z_{1}, z_{2},-\frac{1}{2}\right)$.

Let us consider the term $\operatorname{tr}\left(e\left(u^{\varepsilon}\right)\right)$, whose first term in the expansion (i.e. the $\frac{1}{\varepsilon}$ term) is $u_{3,3}^{0}$. Thus, after integration in the third direction, the two regimes, respectively $\operatorname{tr}\left(e\left(u^{\varepsilon}\right)\right) \geq 0$ and $\operatorname{tr}\left(e\left(u^{\varepsilon}\right)\right) \leq 0$, lead to the conditions $\left[\left[u_{3}^{0}\right]\right] \geq 0$ or $\left[\left[u_{3}^{0}\right]\right] \leq 0$, respectively.

For the first regime (traction), the constitutive equation (2) at the first order of expansion (i.e. $\varepsilon^{0}$ term) gives

$$
\left\{\begin{array}{l}
(\lambda+2 \mu) \hat{u}_{3,3}^{0}=\hat{\sigma}_{33}^{0} \\
\mu \hat{u}_{\alpha, 3}^{0}=\hat{\sigma}_{\alpha 3}^{0}
\end{array}\right.
$$


Because $\hat{\sigma}_{i 3}^{0}$ is independent of $x_{3}$ from (6), we can rewrite these equalities as follows:

$$
\left\{\begin{array}{l}
\hat{u}_{3,3}^{0}=\frac{1}{\lambda+2 \mu} \hat{\sigma}_{33}^{0} \\
\hat{u}_{\alpha, 3}^{0}=\frac{1}{\mu} \hat{\sigma}_{\alpha 3}^{0}
\end{array}\right.
$$

leading, by an integration over the interval $\left[-\frac{1}{2},+\frac{1}{2}\right]$, to

$$
\left\{\begin{array}{l}
{\left[\left[\hat{u}_{3}^{0}\right]\right]=\left(\int_{-\frac{1}{2}}^{\frac{1}{2}} \frac{1}{\lambda\left(x_{3}\right)+2 \mu\left(x_{3}\right)} d x_{3}\right) \hat{\sigma}_{33}^{0}} \\
{\left[\left[\hat{u}_{\alpha}^{0}\right]\right]=\left(\int_{-\frac{1}{2}}^{\frac{1}{2}} \frac{1}{\mu\left(x_{3}\right)} d x_{3}\right) \hat{\sigma}_{\alpha 3}^{0}}
\end{array}\right.
$$

Then, by using the reverse change of variables (see, for example, [5] for more details) and the matching conditions (5), we can return to $\Omega_{ \pm}^{0}$ to obtain the following jump conditions across the interface at order zero

$$
\left\{\begin{array}{l}
\sigma_{33}^{0}=\bar{M}\left[u_{3}^{0}\right] \text { on } \Gamma \\
\sigma_{\alpha 3}^{0}=\bar{\mu}\left[u_{\alpha}^{0}\right] \text { on } \Gamma
\end{array}\right.
$$

which hold on $\Gamma$ when $\left[u_{3}^{0}\right] \geq 0$ and where we have defined the quantities

$$
\left\{\begin{array}{l}
\frac{1}{\bar{M}}=\frac{1}{\varepsilon} \int_{-\frac{\varepsilon}{2}}^{\frac{\varepsilon}{2}} \frac{1}{\lambda\left(x_{3}\right)+2 \mu\left(x_{3}\right)} d x_{3} \\
\frac{1}{\bar{\mu}}=\frac{1}{\varepsilon} \int_{-\frac{\varepsilon}{2}}^{\frac{\varepsilon}{2}} \frac{1}{\mu\left(x_{3}\right)} d x_{3}
\end{array}\right.
$$

with $[h]=\lim _{x_{3} \rightarrow 0^{+}} h\left(x_{1}, x_{2}, x_{3}\right)-\lim _{x_{3} \rightarrow 0^{-}} h\left(x_{1}, x_{2}, x_{3}\right)$ for a given function $h$.

For the second regime (compression), the constitutive equation gives

$$
\hat{u}_{3,3}^{0}=0
$$

and, thus,

$$
\left[\left[\hat{u}_{3}^{0}\right]\right]=0
$$

Then, going back to $\Omega_{ \pm}^{0}$ and using the matching conditions (5), we obtain that

$$
\left[u_{3}^{0}\right]=0
$$

which holds when $\left[u_{3}^{0}\right] \leq 0$.

Using (2) and (11), it can be observed that the following conditions also hold for the second regime:

$$
\left\{\begin{array}{l}
\hat{u}_{1,1}^{0}+\hat{u}_{2,2}^{0}+\hat{u}_{3,3}^{1} \leq 0 \\
\hat{\sigma}_{33}^{0}=\bar{\lambda}\left(\hat{u}_{1,1}^{0}+\hat{u}_{2,2}^{0}+\hat{u}_{3,3}^{1}\right)+2 \mu \hat{u}_{3,3}^{0}=\bar{\lambda}\left(\hat{u}_{1,1}^{0}+\hat{u}_{2,2}^{0}+\hat{u}_{3,3}^{1}\right)
\end{array}\right.
$$

Thus, we find $\hat{\sigma}_{33}^{0} \leq 0$ and using the matching conditions (5), we further obtain that $\sigma_{33}^{0} \leq 0$ on $\Gamma$. 


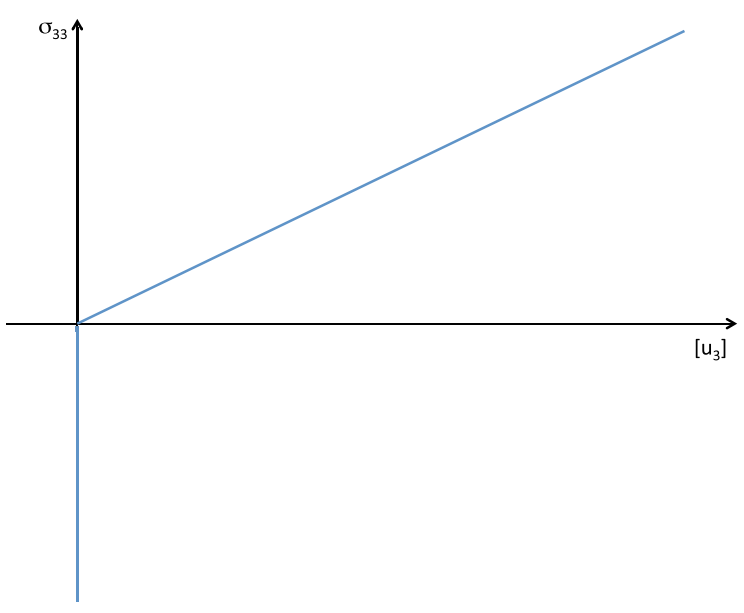

Figure 3. Unilateral contact law obtained by asymptotic expansions

To summarize, unilateral conditions of Signorini type are obtained (see Figure 3) and the approximated interface equilibrium problem at order zero is as follows:

$$
\begin{cases}\operatorname{div} \sigma^{0}+f=0 & \text { in } \Omega_{ \pm}^{0} \\ \sigma^{0} n=g & \text { on } \Gamma_{1} \\ u^{0}=u_{d} & \text { on } \Gamma_{0} \\ \sigma^{0}=A_{ \pm} e\left(u^{0}\right) & \text { in } \Omega_{ \pm}^{0} \\ \sigma^{0} n=C\left[u^{0}\right]_{+}+\tau^{0} & \text { on } \Gamma \\ {\left[u_{3}^{0}\right] \geq 0, \quad \tau_{3}^{0} \leq 0, \quad\left[u_{3}^{0}\right] \tau_{3}^{0}=0} & \text { on } \Gamma\end{cases}
$$

where

$$
\begin{gathered}
C=\left(\begin{array}{ccc}
\bar{\mu} & 0 & 0 \\
0 & \bar{\mu} & 0 \\
0 & 0 & \bar{M}
\end{array}\right) \\
\tau^{0}=\left(\begin{array}{c}
0 \\
0 \\
\tau_{3}^{0}
\end{array}\right)
\end{gathered}
$$

and with

$$
\left[u^{0}\right]_{+}=\left(\begin{array}{c}
{\left[u_{1}^{0}\right]} \\
{\left[u_{2}^{0}\right]} \\
{\left[u_{3}^{0}\right]}
\end{array}\right)
$$

if $\left[u_{3}^{0}\right]>0$,

$$
\left[u^{0}\right]_{+}=\left(\begin{array}{c}
{\left[u_{1}^{0}\right]} \\
{\left[u_{2}^{0}\right]} \\
0
\end{array}\right)
$$

if $\left[u_{3}^{0}\right] \leq 0$.

\section{Finite element implementation and numerical tests}

In this section, we propose a numerical procedure to solve problem (15). For the classical equations of elasticity in $\Omega_{ \pm}^{0}$, a standard finite element method is used. 


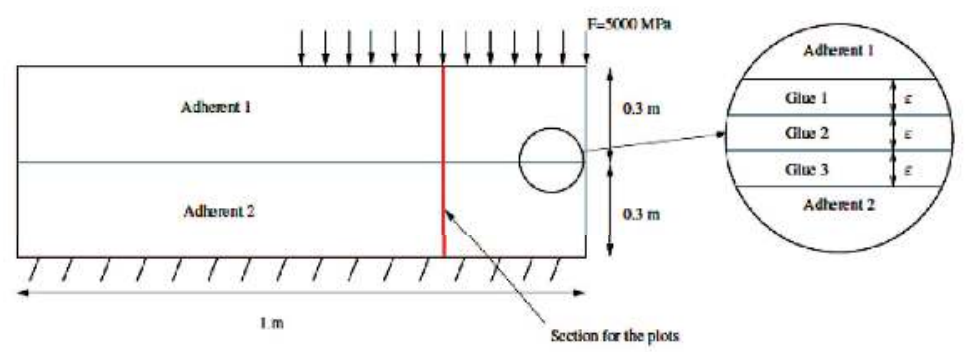

Figure 4. Geometry of the problem ( $\varepsilon=0 \mathrm{~m}$ for the interface problem)

Nevertheless, in order to take into account the jump conditions across the interface $\Gamma$, it is necessary to consider a test function $v$ that can be discontinuous across the interface $\Gamma$. Multiplying the contact condition by the jump of this test function and integrating this relation among the interface, one obtains

$$
\int_{\Gamma}[v] \sigma^{0} n d s=\int_{\Gamma}[v] C\left[u^{0}\right] d s
$$

On the other hand, on each subdomain $\Omega_{-}^{0}$ and $\Omega_{+}^{0}$, we have

$$
\int_{\Omega_{ \pm}^{0}} e(v) A_{ \pm} e\left(u^{0}\right) d s+\int_{\partial \Omega_{ \pm}^{0}} v \sigma^{0} n d s=0
$$

Then adding the three last equalities (on $\Gamma, \Omega_{-}^{0}$, and $\Omega_{+}^{0}$ ), the weak formulation of problem (15) becomes

$$
\int_{\Omega_{ \pm}^{0}} e(v) A_{ \pm} e\left(u^{0}\right) d s+\int_{\Gamma_{1}} v g d s+\int_{\Gamma}[v] C\left[u^{0}\right] d s=0
$$

Finally, using a standard finite element method where the test functions are discontinuous across the interface, it is possible to write a stiffness matrix of problem (20) that is invertible and with standard error estimates (see, for example, [13-15] for more details).

\section{Numerical study}

In this section, we propose a numerical study to assess the influence of the stiffness distribution in the glue on the overall structural behavior. For that purpose, we consider a two-dimensional problem, derived from the three-dimensional problem using plane strain assumptions. In view of these considerations, the interface is now orthogonal to the second direction $x_{2}$ and the interface law (16) remains the same (see, for example, [13]). A solution of the mechanical problem described in the previous section for a particular stiffness distribution in the glue is represented in Figure 5. The mesh is realized using the software GMSH [16].

In all the simulations below, the two adherents have the same material properties, with the Young's modulus equal to $E=20,000 \mathrm{MPa}$ and the Poisson's ratio equal to $v=0.4$.

The adhesive consists of three phases with different glues, characterized by diverse stiffness and varying according to the numerical simulations.

The thickness of each phase is equal to $\varepsilon=0.01 \mathrm{~m}$.

The geometry of the mechanical problem is represented in Figure 4 and an example of solution is shown in Figure 5.

In the simulations hereinafter, the subscript $\varepsilon$ represents the solution of equilibrium problem (1) computed considering the interphase of thickness proportional to $\varepsilon$, whereas the subscript "soft, 0 " represent the solution of equilibrium problem (15) computed using the interface approximation at order zero developed in the present paper.

For the simulations considering the real three-phase interphase, the finite element mesh contains 42,803 nodes and 21,314 finite elements (the total number of degrees of freedom is equal to 85,468), whereas for the simulations with the interface law, the mesh consist of 11,186 elements and 22,530 nodes (the number of degrees of freedom is equal to 44,922). 


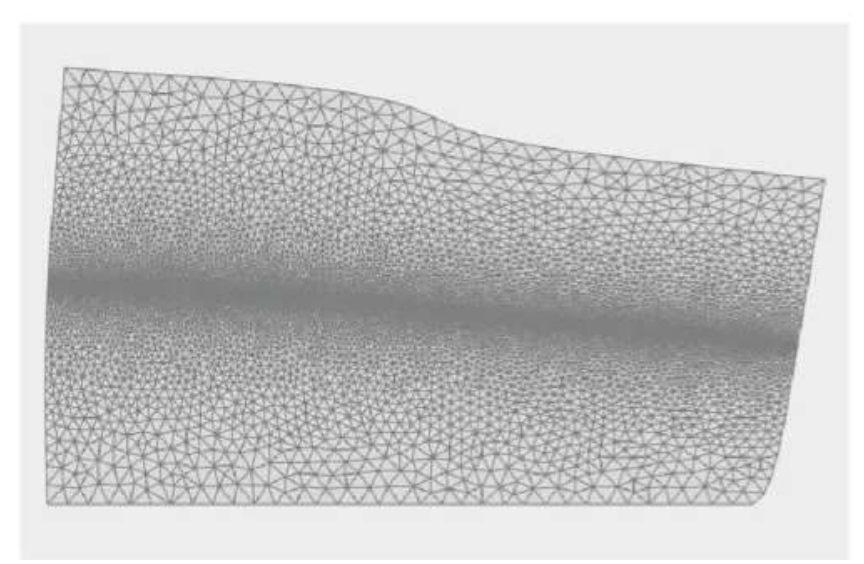

Figure 5. An example of a deformed structure, obtained considering the interphase problem made of three different phases

Table I. Mechanical properties of the three phases of the interphase

\begin{tabular}{lll}
\hline & Young's modulus & Poisson's ratio \\
\hline Glue I & $2000 \mathrm{MPa}$ & 0.4 \\
Glue 2 & $500 \mathrm{MPa}$ & 0.4 \\
Glue 3 & $100 \mathrm{MPa}$ & 0.4 \\
\hline
\end{tabular}
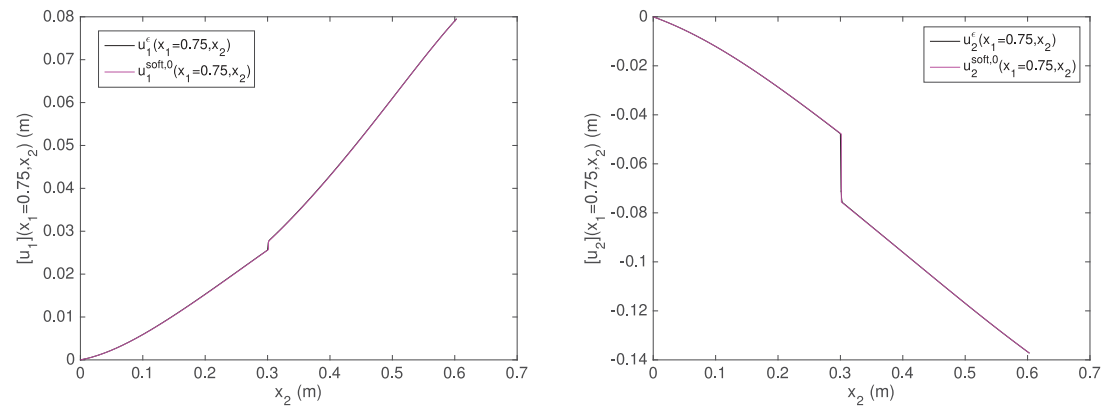

Figure 6. Displacements on the section (left, $u_{1}$; right, $u_{2}$ ): case of small and monotone distribution of rigidities for the glues

\section{I. Test I (small rigidities for the glues)}

In this first set of simulations, we consider an interface composed of three phases, i.e. consisting of three glues with different stiffnesses that are all very small when compared with the stiffness of the adherents.

5.I.I. Results with increasing rigidities In this section, we consider a three phases interphase with a monotone distribution of rigidities, which are from 10 to 200 times smaller than those of the adherents (see Table 1).

In the following figures, the displacements (Figure 6) and the traction (Figure 7) fields on the section drawn in Figure 4 are represented. In Figures 8 and 9, the jumps of the displacements and of the traction along the interface $\Gamma$ are represented.

We can observe that, both in terms of displacements (cf. Figure 6) and in terms of stress (cf. Figure 7), the interface law is a very good approximation and correctly reproduces the overall behavior of the structure. More precisely, the relative error between $u^{\varepsilon}$ and $u^{s o f t, 0}$ inside the adherents, defined by

$$
\mathcal{E}=\frac{\left\|u^{\varepsilon}-u^{\text {soft }, 0}\right\|_{L^{2}\left(\Omega_{ \pm}^{\varepsilon}\right)}}{\left\|u^{\varepsilon}\right\|_{L^{2}\left(\Omega_{ \pm}^{\varepsilon}\right)}}
$$

is equal to $\mathcal{E}=3.65 \times 10^{-3}$.

Figures 6 and 8 show that the jumps in the displacements across the interface $\left[u^{\varepsilon}\right]_{\varepsilon}:=u^{\varepsilon}\left(x_{1}, \frac{\varepsilon}{2}\right)-u^{2}\left(x_{1},-\frac{\varepsilon}{2}\right)$ and $\left[u^{s o f t, 0}\right]$ are numerically very close. 

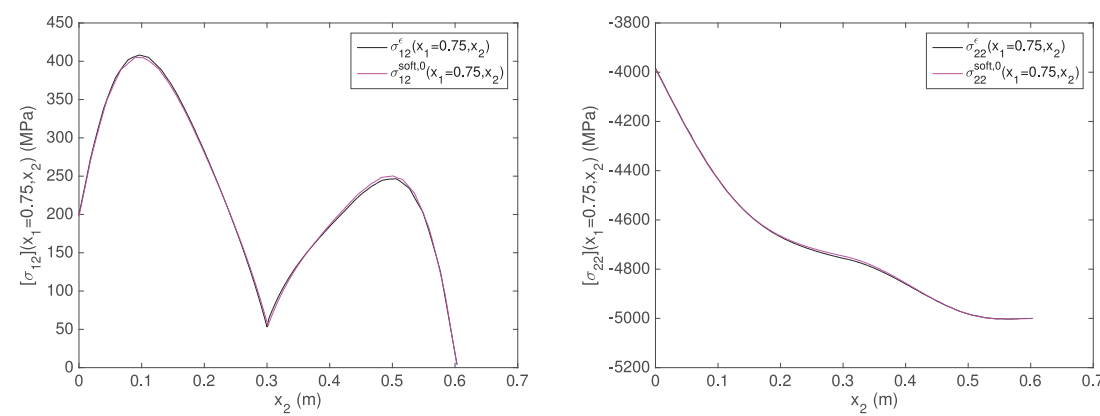

Figure 7. Stresses on the section (left, $\sigma_{12}$; right, $\sigma_{22}$ ): case of small and monotone distribution of rigidities for the glues
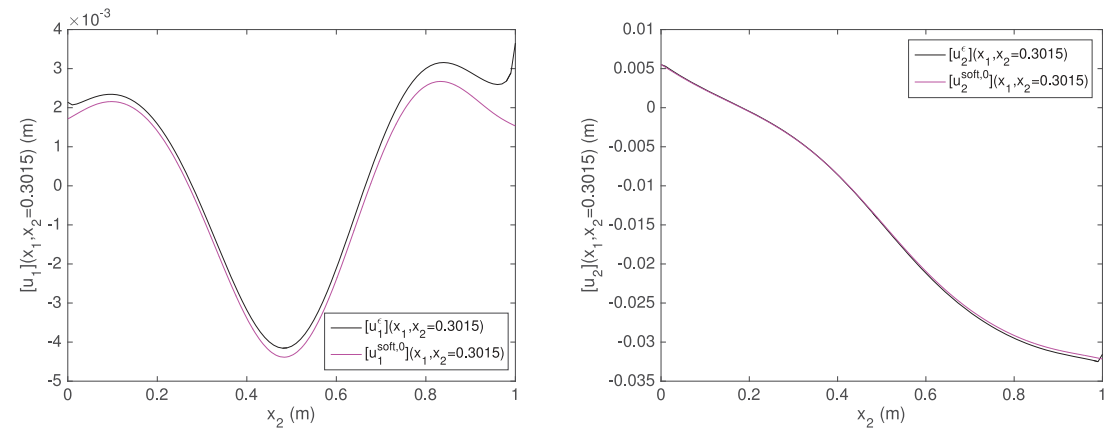

Figure 8. Jumps in the displacements across the interface (left $\left[u_{1}\right]$; right, $\left.\left[u_{2}\right]\right)$ : case of small and monotone distribution of rigidities for the glues
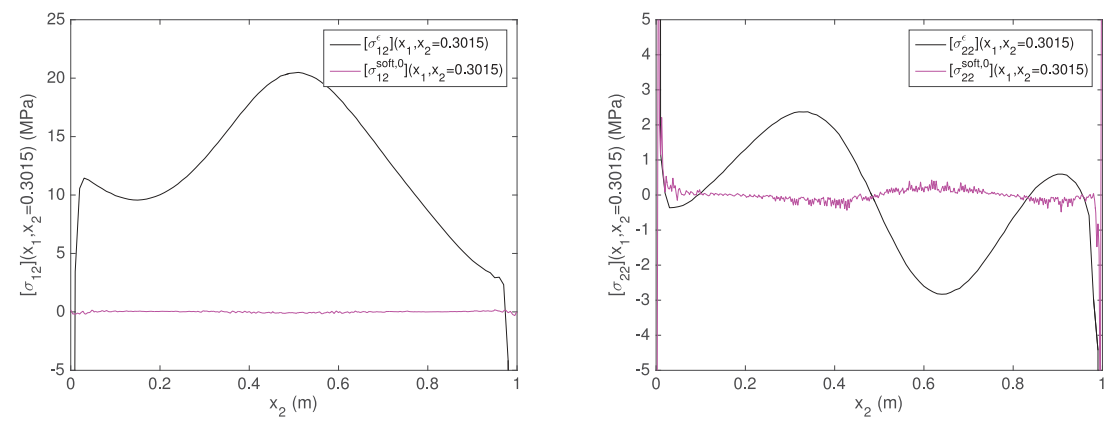

Figure 9. Jumps of the traction across the interface (left, $\left[\sigma_{12}\right]$; right, $\left[\sigma_{22}\right]$ ): case of small and monotone distribution of rigidities for the glues

According to equation (6), the jumps in the stresses $\left[\sigma_{12}^{\text {soft }, 0}\right]$ and $\left[\sigma_{22}^{\text {soft }, 0}\right]$ have to be equal to zero across the interface. This is reproduced by our simulations, as can be observed in Figure 9. Consequently, the proposed finite element method is appropriated, and in the case of a very small rigidity of the glue in the interphase, the approximation that leads to considering vanishing jumps in the constraint is justified. for $\sigma_{22}$.

Indeed, the ratio $\frac{\max \left\{\left[\sigma_{i j}^{0}\right]\left(x_{1}, x_{2}\right),\left(x_{1}, x_{2}\right) \in \Gamma\right\}}{\max \left\{\left|\sigma_{i j}^{0}\right|\left(x_{1}, x_{2}\right),\left(x_{1}, x_{2}\right) \in \Omega_{ \pm}^{0}\right\}}$ is equal to $5 \times 10^{-3}$ for the shear stress $\sigma_{12}$, and is equal to $7.5 \times 10^{-3}$

5. I.2. Results for a non-monotone stiffness distribution. In this section, we consider a three-phase interphase with stiffnesses much smaller than those of the adherents but now with a non-monotone distribution, given in Table 2.

In Figures 10-13, we present some comparisons between the results obtained considering the three-phase problem (1) and its approximated problem (15) using jumps in displacement across the interface $\Gamma$.

In Figures 10 and 11, one can observe that the overall response of the structure is very similar to the case of a monotone stiffness distribution in the interphase. The jumps, both in terms of displacements (Figure 12) and 
Table 2. Mechanical properties of the three phases composing the interphase

\begin{tabular}{lll}
\hline & Young's modulus & Poisson's ratio \\
\hline Glue I & $2000 \mathrm{MPa}$ & 0.4 \\
Glue 2 & $100 \mathrm{MPa}$ & 0.4 \\
Glue 3 & $500 \mathrm{MPa}$ & 0.4 \\
\hline
\end{tabular}
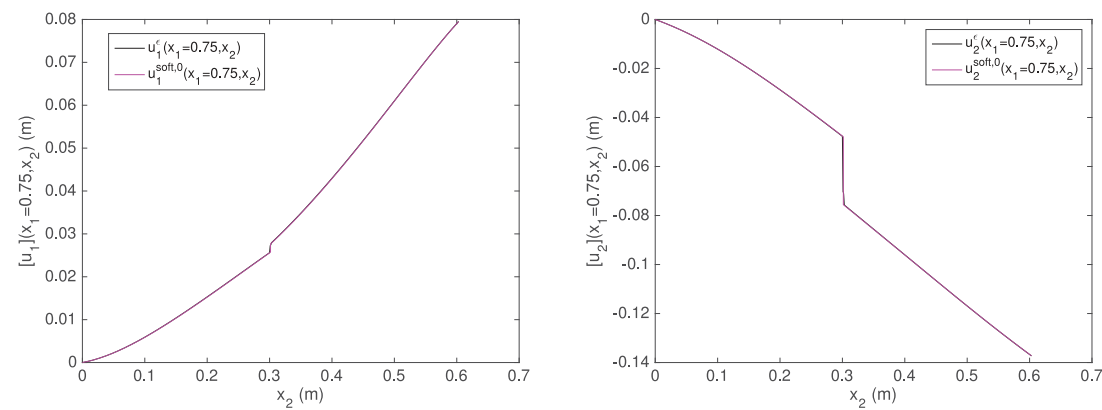

Figure 10. Displacements on the section (left, $u_{1}$; right, $u_{2}$ ): case of small and non-monotone distribution of rigidities for the glues
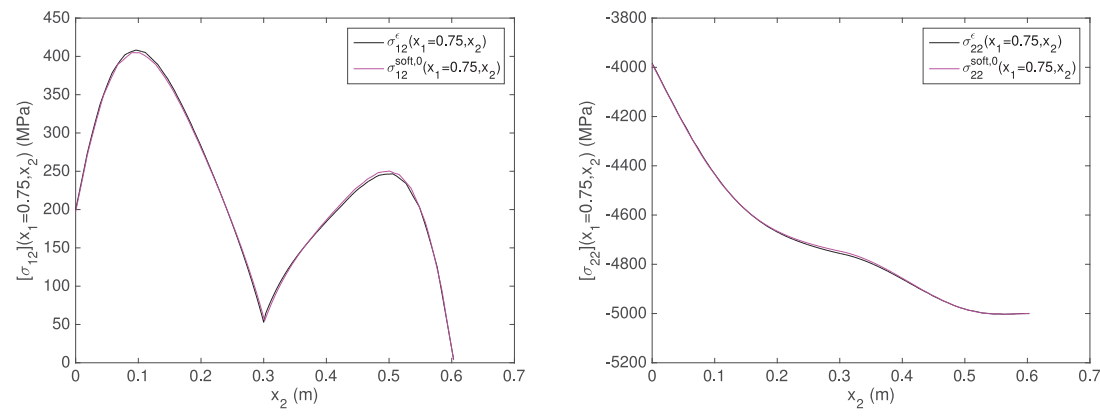

Figure I I. Stresses on the section (left, $\sigma_{12}$; right, $\sigma_{22}$ ): case of small and non-monotone stiffness distribution for the glues
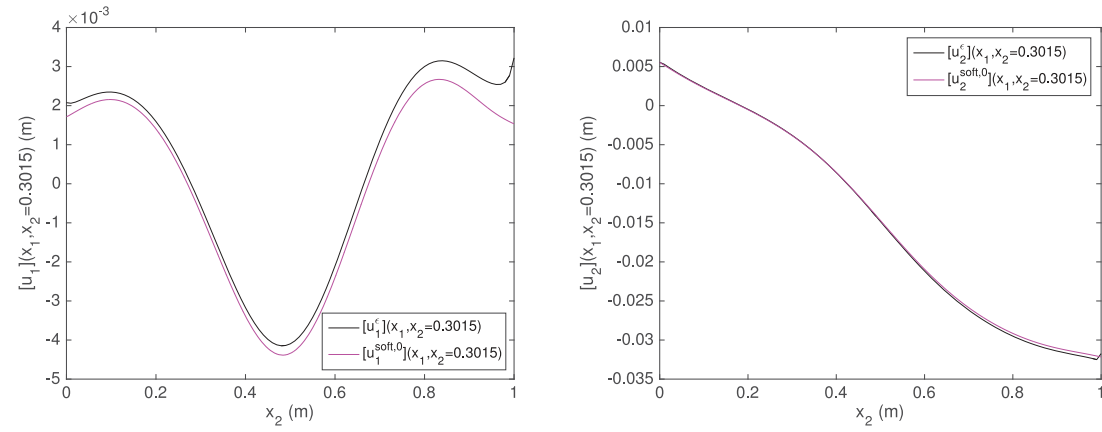

Figure 12. Jumps in the displacements across the interface (left, $\left[u_{1}\right]$; right, $\left[u_{2}\right]$ ): case of small and non-monotone stiffness distribution for the glues

stresses (Figure 13), are also very close for the two cases. As a consequence, the interface law given by formula (10) can be considered to provide a good approximation of the behavior of the structure.

5. I.3. Comparison of results for monotone and non-monotone stiffness distributions. We compare here the results obtained with both monotone and non-monotone stiffness distribution in the interphase. For that purpose, we present in Figures 14 and 15 an enlarged view of the displacements and the stresses calculated in the proximity of the interphase, respectively.

One can observe that we obtain very similar results for both monotone and non-monotone stiffness distribution in the glues, and the interface condition provides a very good approximation of the jumps both for the 

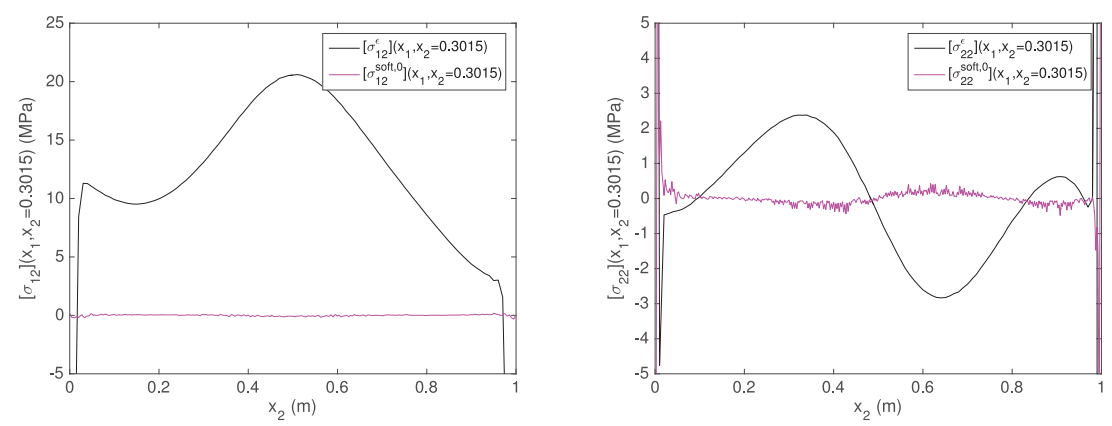

Figure 13. Jumps in the constraints across the interface (left, [ $\left.\sigma_{12}\right]$; right, [ $\left.\sigma_{22}\right]$ ): case of small and non-monotone stiffness distribution for the glues
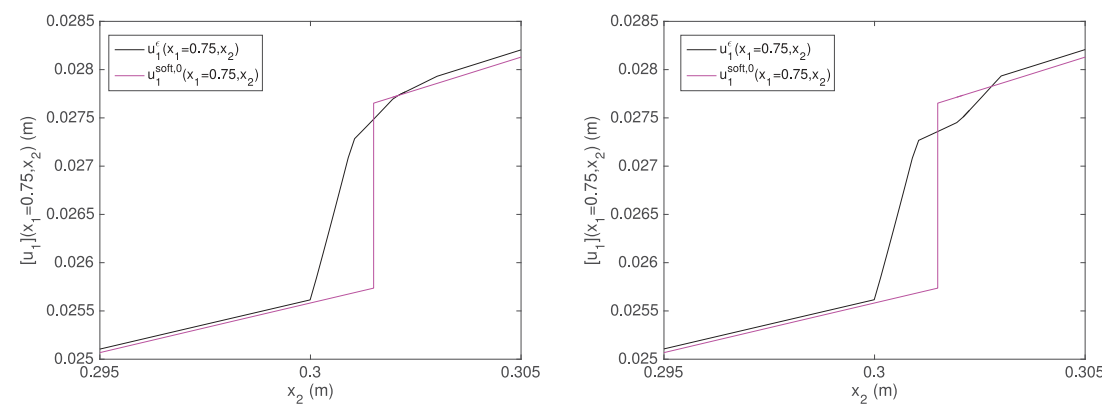

Figure 14. Displacements $u_{1}$ at the interface on the section for monotone (left) and non-monotone (right) increasing stiffness
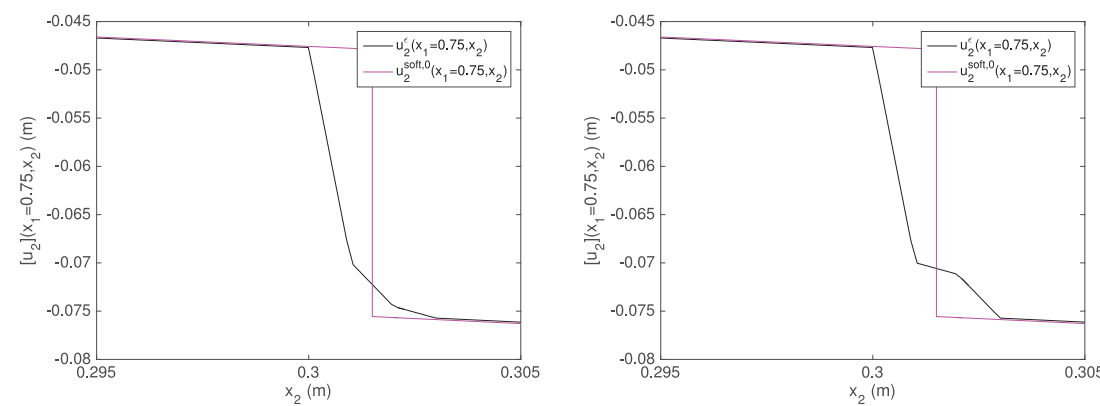

Figure I5. Displacements $u_{2}$ at the interface on the section for monotone (left) and non-monotone (right) increasing stiffness

displacement and the stress components. In the case of a non-monotone stiffness distribution, the relative error between $u^{\varepsilon}$ and $u^{\text {soft, } 0}$ is equal to $\mathcal{E}=3.43 \times 10^{-3}$, which is very close to the value obtained in the case of a monotone stiffness distribution.

\subsection{Test 2 (very different stiffnesses for the materials composing the glue)}

In this section, we present the results for an interphase in which the stiffness is strongly varying, from values close to the stiffness of the adherents to much smaller values.

In this case, we compare results for both a monotone (see Table 3) and a non-monotone (see Table 4) stiffness distribution in the interphase.

Let us note that, as in the previous example, the interface law is calculated in the same way for all cases, being given only by an average using formula (10).

Figures 16, 17, and 18 show enlarged views of the displacements $u_{1}$ and $u_{2}$ and the shear stress $\sigma_{12}$ in the proximity of the interphase. The jumps in the displacements $\left[u^{\varepsilon}\right]_{e}$ at the interphase are very similar for the two cases, even if we can appreciate the differences in the stiffness distribution inside the interphases (black curves in Figures 16 and 17). Note that these jumps are correctly approximated by the interface law (red curves) and that $\left[u^{\varepsilon}\right]_{e}$ and $\left[u^{s o f t, 0}\right]$ are very close. 
Table 3. Mechanical properties of the three phases composing the interphase: case of a monotone stiffness distribution in the interphase

\begin{tabular}{lll}
\hline & Young's modulus & Poisson's ratio \\
\hline Glue I & $10,000 \mathrm{MPa}$ & 0.4 \\
Glue 2 & $5000 \mathrm{MPa}$ & 0.4 \\
Glue 3 & $1000 \mathrm{MPa}$ & 0.4 \\
\hline
\end{tabular}

Table 4. Mechanical properties of the three phases of the interphase: case of a non-monotone stiffness distribution in the interphase

\begin{tabular}{lll}
\hline & Young's modulus & Poisson's ratio \\
\hline Glue I & $10,000 \mathrm{MPa}$ & 0.4 \\
Glue 2 & $1000 \mathrm{MPa}$ & 0.4 \\
Glue 3 & $5000 \mathrm{MPa}$ & 0.4 \\
\hline
\end{tabular}
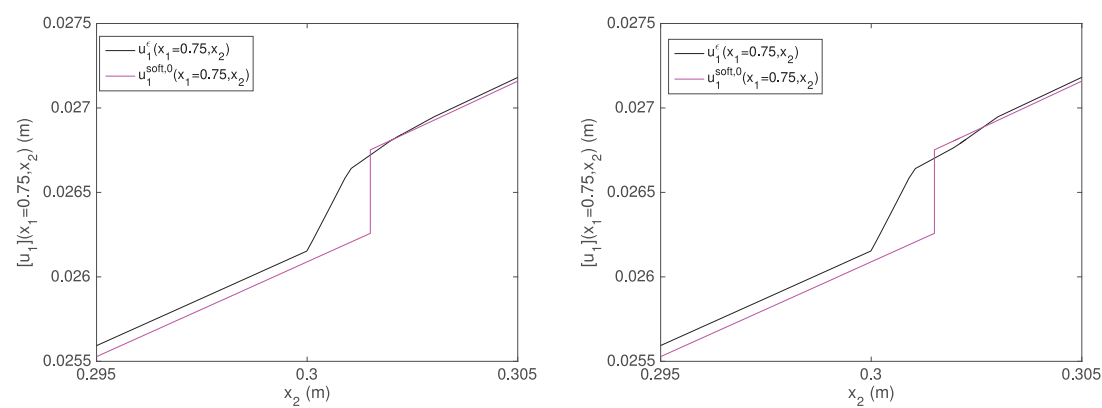

Figure 16. Enlarged view of the displacements $u_{1}$ at the interface on the section for monotone (left) and non-monotone (right) increasing stiffness distributions
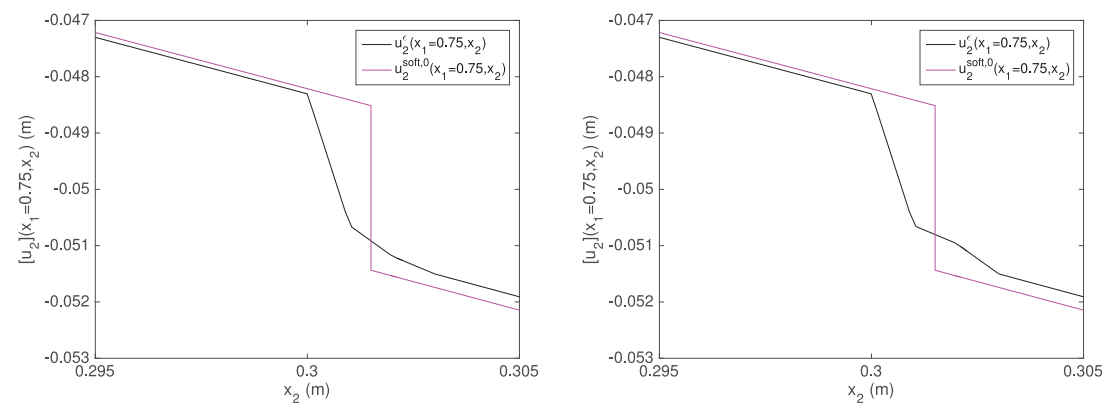

Figure 17. Enlarged view of the displacements $u_{2}$ at the interface on the section for monotone (left) and non-monotone (right) increasing stiffness distributions
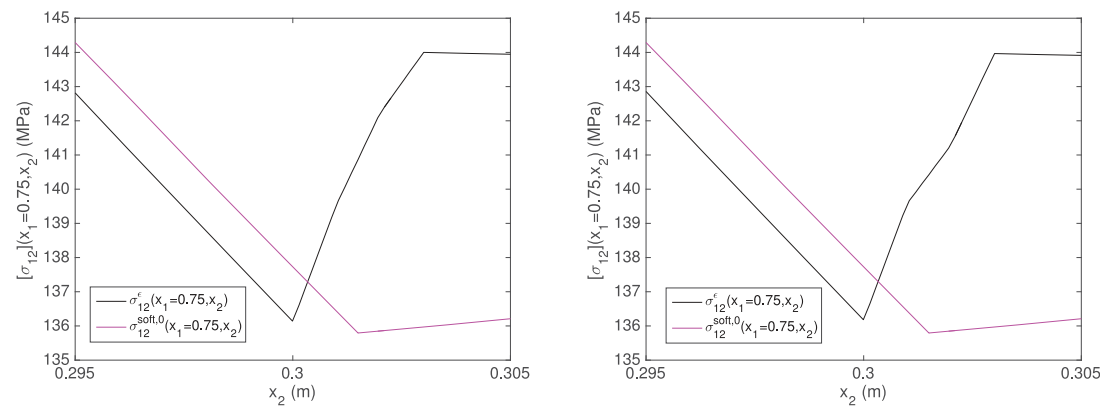

Figure 18. Enlarged view of the shear stress $\sigma_{12}$ at the interface on the section for monotone (left) and non-monotone (right) increasing stiffness distributions 
Table 5. Example with five glues in the interface: the monotone case

\begin{tabular}{lll}
\hline & Young's modulus & Poisson's ratio \\
\hline Glue I & $1000 \mathrm{MPa}$ & 0.4 \\
Glue 2 & $1000 \mathrm{MPa}$ & 0.4 \\
Glue 3 & $1000 \mathrm{MPa}$ & 0.4 \\
Glue 4 & $10,000 \mathrm{MPa}$ & 0.4 \\
Glue 5 & $10,000 \mathrm{MPa}$ & 0.4 \\
\hline
\end{tabular}

Table 6. Example with five glues in the interface: the non-monotone case

\begin{tabular}{lll}
\hline & Young's modulus & Poisson's ratio \\
\hline Glue I & $1000 \mathrm{MPa}$ & 0.4 \\
Glue 2 & $10,000 \mathrm{MPa}$ & 0.4 \\
Glue 3 & $1000 \mathrm{MPa}$ & 0.4 \\
Glue 4 & $10,000 \mathrm{MPa}$ & 0.4 \\
Glue 5 & $1000 \mathrm{MPa}$ & 0.4 \\
\hline
\end{tabular}
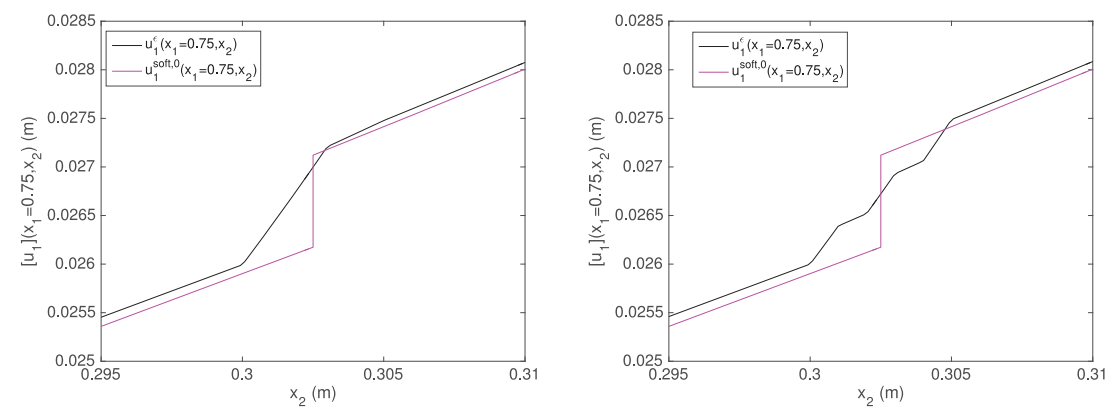

Figure 19. Enlarged view of the displacements $u_{1}$ at the interface on the section for monotone (left) and non-monotone (right) increasing rigidity for interfaces made of five different glues

The overall response of the structure is also well approximated, with a relative difference between $u^{\varepsilon}$ and $u^{\text {soft }, 0}$ equal to $\mathcal{E}=3.07 \times 10^{-3}$ for the case of a monotone stiffness distribution, and is equal to $\mathcal{E}=3.09 \times 10^{-3}$ for the case of a non-monotone stiffness distribution.

Concerning the shear stress, one can observe that the jumps at the interphase are similar for monotone and non-monotone distribution. Moreover, the ratio between the jumps in the shear stress and the mean value of the shear stress around the interface is comparable to the previous ones and equal to $4 \times 10^{-2}$. Note also that in this test the jumps in the stress component $\sigma_{22}$, which is not represented here, is of the same order as that obtained in the previous test.

\subsection{Test 3 (example with five different glues)}

We now compare results obtained with two distributions of glues within the interface, provided in Tables 5 and 6 , respectively.

Figures 16, 19, and 21 show similar results for stiffness distributions monotone and non-monotone; moreover, the interface law provides a good approximation of what occurs inside the interphase.

In this set of simulations, the relative difference between $u^{\varepsilon}$ and $u^{\text {soft, } 0}$ is equal to $\mathcal{E}=4.86 \cdot 10^{-3}$ for the case of a monotone stiffness distribution, and is equal to $\mathcal{E}=4.84 \cdot 10^{-3}$ for the case of a non-monotone stiffness distribution.

\subsection{Test 4: an example with traction}

In this section, we present an example of a structure with an adhesive composed of three layers and subjected to a traction loading (cf. Figure 22). The mechanical properties of each layer are provided in Table 7. The stiffness distribution is non-monotone. 

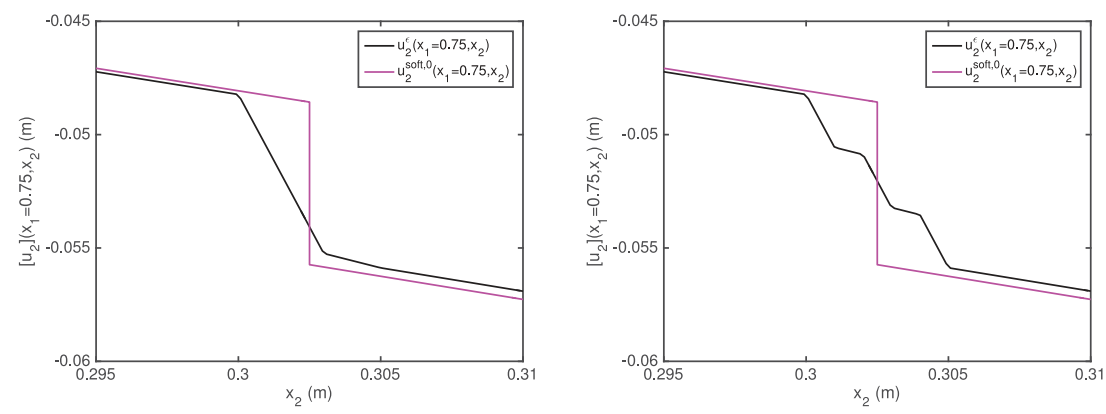

Figure 20. Enlarged view of the displacements $u_{2}$ at the interface on the section for monotone (left) and non-monotone (right) increasing rigidity for interfaces made of five different glues
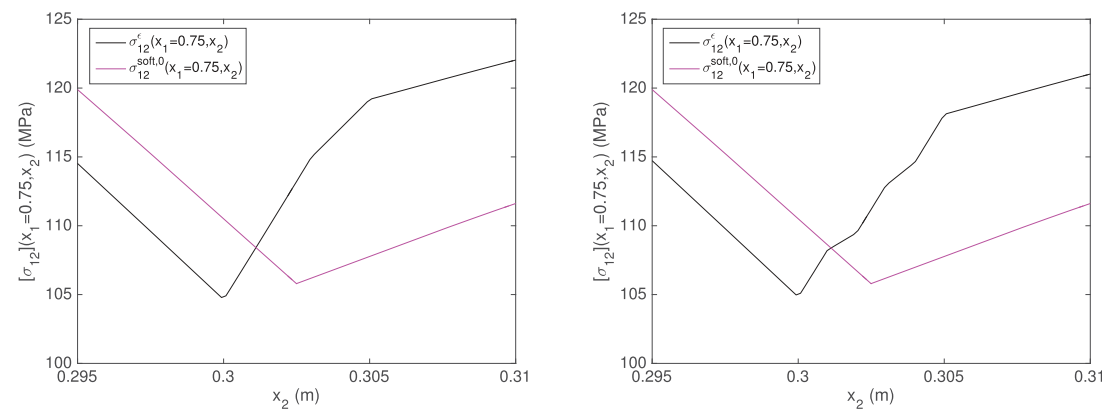

Figure 2I. Enlarged view of the shear stress $\sigma_{12}$ at the interface on the section for monotone (left) and non-monotone (right) increasing rigidity for interfaces made of five different glues

Table 7. Mechanical properties of the three phases composing the interphase: case of a monotone stiffness distribution in the interphase

\begin{tabular}{lll}
\hline & Young's modulus & Poisson's ratio \\
\hline Glue I & $1000 \mathrm{MPa}$ & 0.4 \\
Glue 2 & $10,000 \mathrm{MPa}$ & 0.4 \\
Glue 3 & $5000 \mathrm{MPa}$ & 0.4 \\
\hline
\end{tabular}

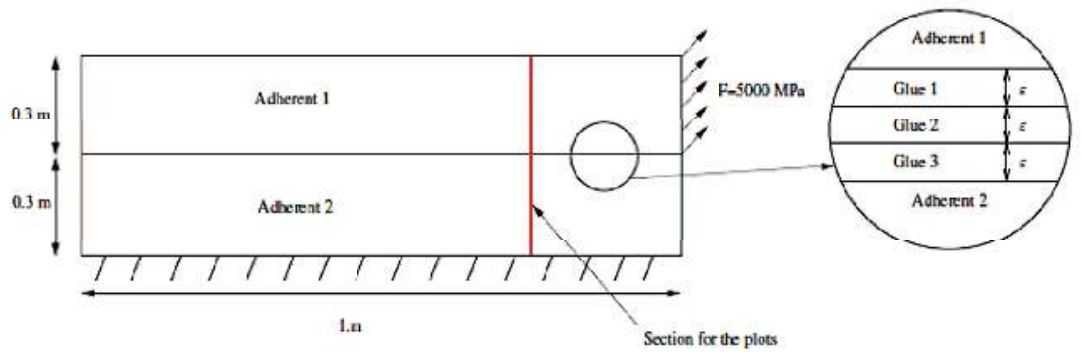

Figure 22. Geometry of the problem ( $\varepsilon=0 \mathrm{~m}$ for the interface problem)

Figure 23 shows an enlarged view of the displacement component $u_{1}$ (left) and the shear stress $\sigma_{12}$ (right), both calculated in close proximity of the interphase. One can observe that, in this situation, the approximation of the multi-layer initial problem provided by the soft interface modeling is correct.

\subsection{Conclusion on the numerical results}

In all the numerical examples presented, one can observe that the interface law given in (10) and in (15) always provides a good approximation of the initial multi-phase problem, even if some of the materials in the interphase are characterized by large values of the elastic modulus. Note that this result is not trivial, because our 

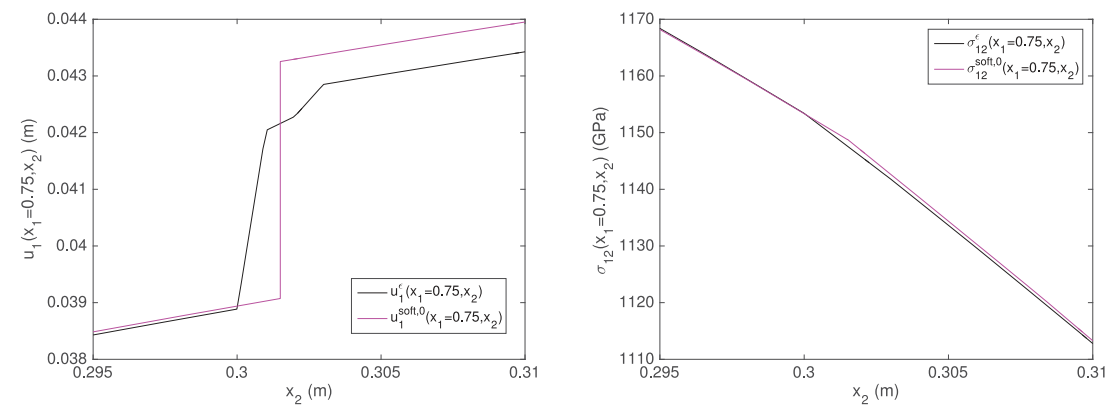

Figure 23. Enlarged view of the displacement component $u_{1}$ (left) of the shear stress $\sigma_{12}$ (right) at the interface on the section for non-monotone stiffness distribution in an interphase made of three different glues and submitted to a traction loading

asymptotic analysis has been based on the assumption that the elastic properties are of order $\varepsilon$. Note also that the modeling with the proposed soft interface law is able to correctly describe the structural behavior of the composite for different stiffness distributions in the interphase (monotone or non-monotone).

\section{Conclusion}

In this paper, we have presented a study of a composite made of three elastic bodies: two adherents and a thin adhesive. The elastic properties of the adhesive are assumed to vary along the thickness direction and to be characterized by two regimes, one in traction (soft) and one in compression (hard). After the derivation of an interface law for such a structure, we have numerically compared the response of the initial three-phase composite with those of an approximating structure made of two phases joined by a material interface described by the calculated interface law. We have shown that the two responses are in good agreement, especially in terms of displacements, for a low thickness of the graded adhesive (with a ratio varying from $0.01 \mathrm{~mm}$ to $0.3 \mathrm{~m}$ ) and for various stiffness distributions in the adhesive. A good agreement has been found even if the elastic moduli of some layers of the adhesive are of the same order as that of the adherents.

These findings clearly indicate that the approach of substituting the small graded interphase with the calculated interface law provides a robust modeling for the composite. This fact, not a priori obvious, represents the main original result of the present paper. Finally, our results also show that the proposed asymptotic analysis is flexible enough to generalize analogous previous theoretical results obtained for standard elastic materials (cf. $[5,6,13]$, for example) to composites realized with FGMs.

\section{Funding}

The author(s) received no financial support for the research, authorship, and/or publication of this article.

\section{References}

[1] Udupa, G, Rao, SS, and Gangadharan, K. Functionally graded composite materials: An overview. Proc Mater Sci 2014; 5: 1291-1299.

[2] Niino, M, Hirai, T, and Watanabe, R. The functionally gradient materials. J Jap Soc Compos Mat 1984; 13: $257-264$.

[3] Abdelmoula, R, Coutris, M, and Marigo, J. Comportement asymptotique d'une interface mince. C R Acad Sci Sér IIB 1998; 326 : 237-242.

[4] Lebon, F, and Rizzoni, R. Asymptotic study of a soft thin layer: the non convex case. Mech Adv Mat Struct 2008; 15 : 12-20.

[5] Lebon, F, and Rizzoni, R. Asymptotic behavior of a hard thin linear interphase: An energy approach. Int J Solids Struct 2011; 48: 441-449.

[6] Lebon, F, and Rizzoni, R. Modelling adhesion by asymptotic techniques. Adhesive Properties in Nanomaterials, Composites and Films 2011; : 96-126.

[7] Licht, C, and Michaille, G. A modeling of elastic adhesive bonded joints. Adv Math Sci Appl 1997; 7: 711-740.

[8] Sanchez-Palencia, E. Non-homogeneous media and vibration theory (Lecture Notes in Physics, vol. 127). Berlin: Spriger-Verlag, 1980 .

[9] Hashin, Z. Thin interphase/imperfect interface in conduction. J Appl Phys 2001; 89: 2261.

[10] Hashin, Z. Thin interphase/imperfect interface in elasticity with application to coated fiber composites. J Mech Phys Solids 2002; 50: 2509-2537. 
[11] Javili, A, Kaessmair, S, and Steinmann, P. General imperfect interfaces. Comput Meth Appl Mech Eng 2014; 275 : 76-97.

[12] Javili, A, Steinmann, P, and Mosler, J. Micro-to-macro transition accounting for general imperfect interfaces. Computer Methods in Applied Mechanics and Engineering 2017; 317: 274-317.

[13] Dumont, S, Lebon, F, and Rizzoni, R. An asymptotic approach to the adhesion of thin stiff films. Mech Res Commun 2014; 58: 24-35.

[14] Lebon, F, Khaoua, AO, and Licht, C. Numerical study of soft adhesively bonded joints in finite elasticity. Comput Mech 1997; 21: $134-140$.

[15] Nairn, J. Numerical implementation of imperfect interfaces. Computational Materials Science 2007; 40: 525-536.

[16] Geuzaine, C, and Remacle, JF. GMSH: a three-dimensional finite element mesh generator with built-in pre- and post-processing facilities. Int J Numer Meth Eng 2009; 679: 1309-1331. 